\title{
Appendix Neuroendocrine Tumor G1
}

National Cancer Institute

\section{Source}

National Cancer Institute. Appendix Neuroendocrine Tumor G1. NCI Thesaurus. Code C4138.

A well differentiated, low grade tumor with neuroendocrine differentiation that arises from the appendix. The mitotic count is less than 2 per $10 \mathrm{HPF}$ and/or the Ki67 index is equal to or less than 2 percent. 